\title{
Metastatic Neuroendocrine Tumor in the Thyroid: Report of an Incidental Diagnosis
}

\author{
Uzma Mohammad Siddiqui $^{\mathrm{a}, \mathrm{c}}$, Aida L. Valencia-Guerrero ${ }^{\mathrm{b}}$, \\ Nina Rosano ${ }^{\mathrm{a}}$, Eyad Hamoudeh ${ }^{\mathrm{a}}$
}

\begin{abstract}
Secondary tumors of the thyroid are quite rare with an estimated prevalence of $<1 \%$. Herein, we describe the incidental discovery of metastatic small cell lung cancer in a non-toxic multinodular goiter. A 57-year-old lady has had a non-toxic multinodular goiter for over 10 years. She has had two thyroid fine needle aspirations (FNAs) in the past, which were reported benign. As the goiter was increasing in size and causing compression, she underwent a total thyroidectomy. Pathology revealed multi-centric high grade carcinoma with small cell/neuroendocrine features with the largest focus being $1.1 \mathrm{~cm}$. Calcitonin staining was negative. Further imaging revealed a $68 \times 37$ $\times 51 \mathrm{~mm}$ soft tissue mediastinal mass and an $18 \times 10 \mathrm{~mm}$ left upper lobe pulmonary nodule. She was then initiated on chemotherapy and radiation therapy for extensive stage small cell cancer of the lung. After two cycles of chemotherapy, restaging chest computed tomography $(\mathrm{CT})$ showed interval improvement in lung masses. This rare presentation of small cell lung cancer with multi-centric metastasis to the thyroid highlights the need to obtain nodule directed FNAs, as the two non-directed FNAs done prior to surgery were benign. It also points out to clinicians that rapidly growing goiters are a cause for concern, and should be evaluated, even if patients are not symptomatic.
\end{abstract}

Keywords: Thyroid; Neuroendocrine; Metastatic; Goiter

\section{Introduction}

According to the National Cancer Institute, there were over half a million people living with thyroid cancer in the United

\footnotetext{
Manuscript submitted November 3, 2017, accepted November 15, 2017

aDepartment of Endocrinology, Diabetes \& Metabolism, University of Massachusetts Medical School, 55 Lake Avenue North, Worcester, MA 01655, USA bepartment of Pathology, University of Massachusetts Medical School, One Innovation Drive, Three Biotech, Worcester, MA, USA

${ }^{\mathrm{c} C}$ Corresponding Author: Uzma Mohammad Siddiqui, Department of Endocrinology, Diabetes \& Metabolism, University of Massachusetts Medical School, 55 Lake Avenue North, Worcester, MA 01655, USA.

Email: uzma.siddiqui@umassmemorial.org
}

doi: https://doi.org/10.14740/jem468w
States as of 2014. Medullary thyroid cancer, which originates from the parafollicular cells, is the most common neuroendocrine tumor of the thyroid, accounting for about $2 \%$ of all thyroid cancer. Aside from these, secondary tumors of the thyroid are quite rare. Studies have shown that the prevalence of metastatic disease to the thyroid in patients who have undergone thyroidectomies is less than $1 \%$, although a higher prevalence of up to $9 \%$ has been recorded in autopsy studies [1]. Of all secondary tumors of the thyroid, metastases from the lungs are most common [2]. Other tumors that have been noted to have metastasized to the thyroid include breast, esophagus, stomach and kidneys $[1,3]$. Often, metastasis to the thyroid is an indicator of widespread disease and most patients have a short survival time thereafter [4].

Herein, we describe the incidental discovery of metastatic small cell lung cancer in a large non-toxic multinodular goiter.

\section{Case Report}

The patient is a 57-year-old lady who has had a non-toxic multinodular goiter for over 10 years. She did not have any known hypothyroidism and no radiation exposure. In October 2016, she had two non-directed thyroid fine needle aspirations (FNAs), one from the left and one from the right side, which were benign and consistent with lymphocytic thyroidi-

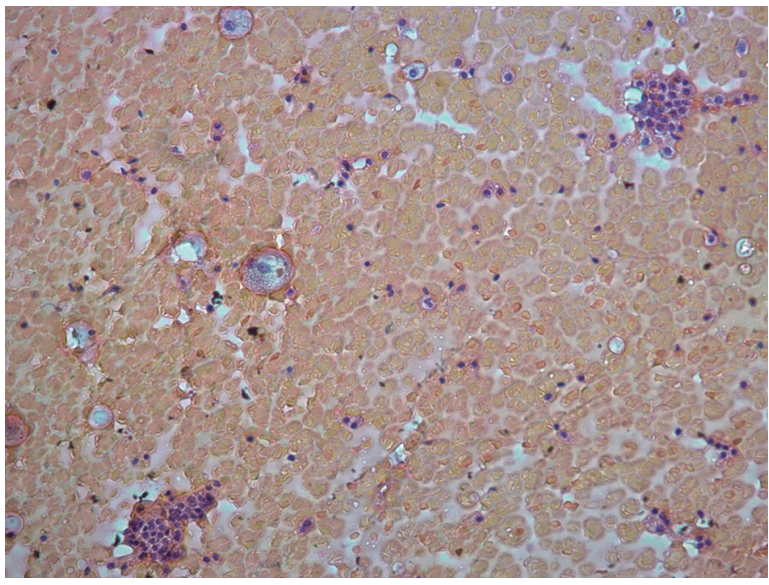

Figure 1. Cytology from thyroid fine needle aspiration. This shows benign follicular cells with increased lymphocytes in the background and scattered macrophages $(\times 400)$. 


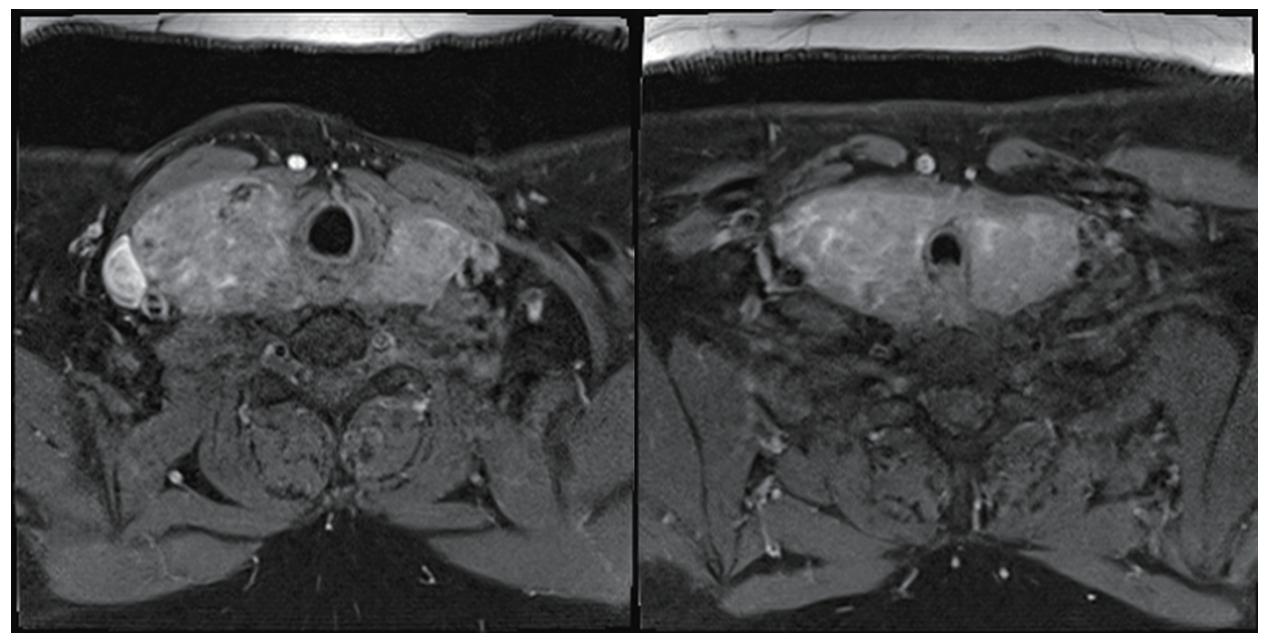

Figure 2. MRI of face and neck. Bilaterally enlarged thyroid lobes, right more than left, laterally deviating carotid arteries and jugular veins.

tis (Fig. 1). Her other medical history was pertinent for type 2 diabetes mellitus, hypertension and hyperlipidemia. She was an active smoker, and there was no thyroid disease in the family.

The patient's primary care physician noted that the thyroid gland was growing, and therefore obtained a magnetic resonance imaging (MRI) which revealed deviated carotid arteries bilaterally because of the thyroid as well as narrowing of the trachea and the esophagus (Fig. 2). Although the patient was asymptomatic, due to the increasing size of the goiter on imaging, she was referred for a total thyroidectomy in February 2017. Pathology from the right thyroid (weight: $130 \mathrm{~g}$ ) revealed multi-centric high grade carcinoma with the largest focus being $1.1 \mathrm{~cm}$. Margins were negative and no extrathyroidal extension or lympho-vascular invasion was noted. Microscopic examination showed multi-centric solid sheets and nests of neoplastic cells with neuroendocrine features (Fig. 3). The tumor cells were positive for chromogranin and synapto-

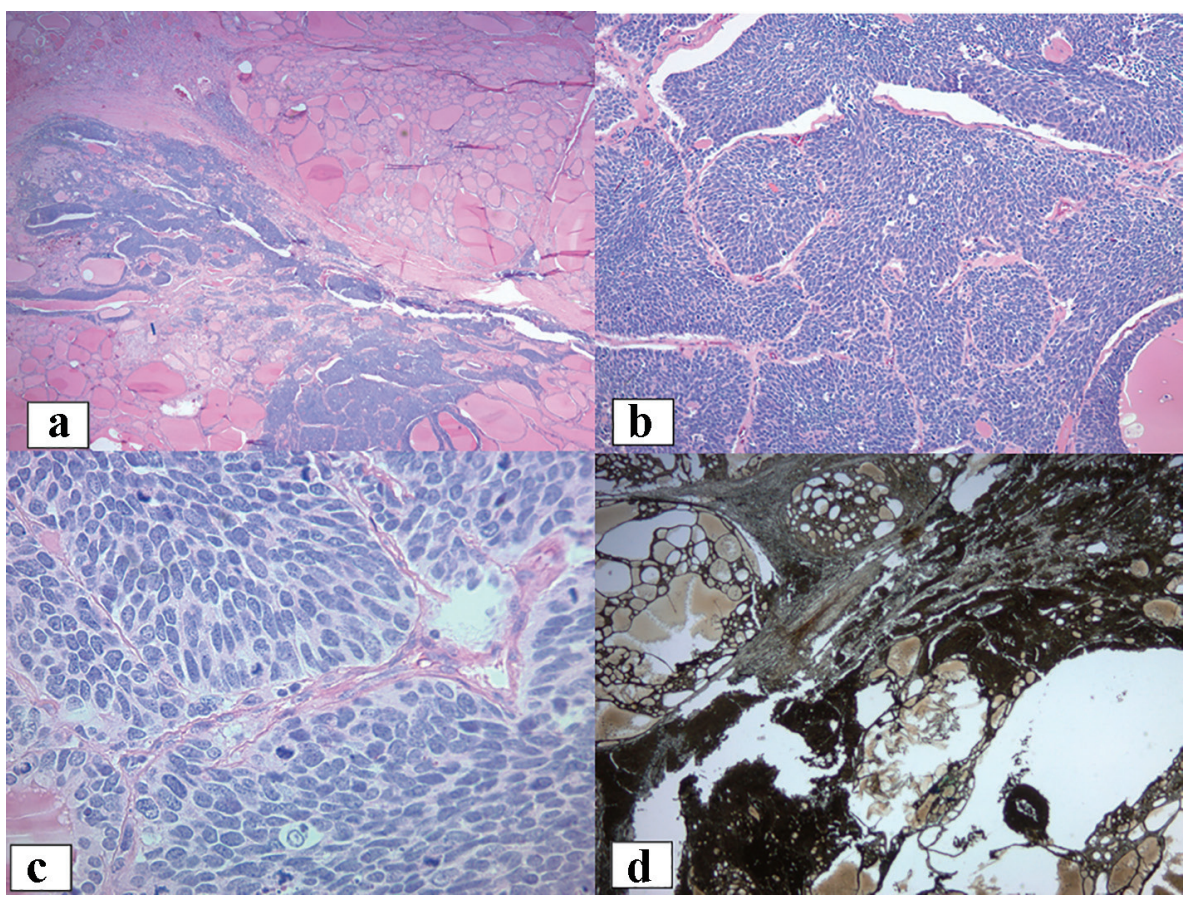

Figure 3. Histologic features of the thyroid. $(a, b)$ These images are in medium power view showing solid sheets and nests of carcinoma in a background of a benign multinodular goiter $(\times 100, \times 200)$. (c) The tumor cells contain clear to eosinophilic cytoplasm and oval nuclei with fine dispersed, granular chromatin typical of neuroendocrine tumors. Nuclear molding as well as numerous mitoses is present, including atypical forms (× 400). (d) CD-56 immunohistochemistry shows diffuse and strong cytoplasmic staining. 


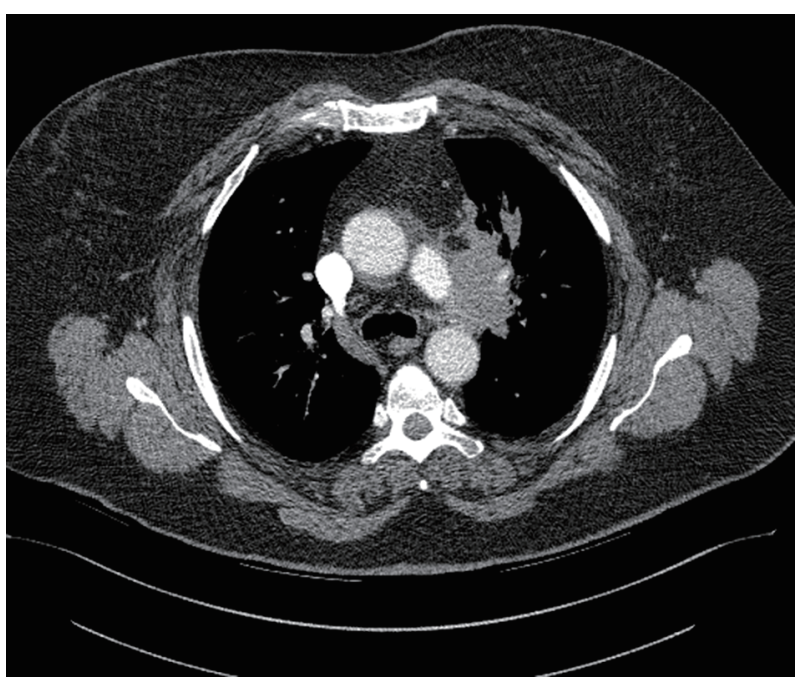

Figure 4. Chest CT. A $68 \times 37 \times 51 \mathrm{~mm}$ soft tissue mass is seen within the mediastinum pre vascular space and exhibits mass effect.

physin (weak), CD56 (strong and diffuse), pan-cytokeratin, low molecular weight keratin (dot like pattern), TTF1, mCEA and showed a proliferation index of $80 \%$ (Ki-67). Other stains such as CK7, CK20, thyroglobulin, calretinin, PAX8, p40, CDX2, calcitonin, mammaglobin, GATA3, and S100 were negative.

The morphology and immunophenotype was most consistent with a high-grade carcinoma with small cell/neuroendocrine features. The left lobe and the isthmus (weight: $52 \mathrm{~g}$ ) revealed multinodular hyperplasia but no carcinoma was identified.

Laboratory workup showed a calcitonin level of $<2 \mathrm{pg} /$ $\mathrm{mL}(\leq 5)$ and a carcinoembryonic antigen was $6.4 \mathrm{ng} / \mathrm{mL}(8 \%$ of smokers: 5.1 - 10). This was followed with imaging of patient's chest, abdomen and pelvis which revealed a $68 \times 37$ $\times 51 \mathrm{~mm}$ soft tissue mediastinal mass partially encasing the left main pulmonary artery and left superior pulmonary vein (Fig. 4). It also showed an adjacent $18 \times 10 \mathrm{~mm}$ left upper lobe pulmonary nodule. Another $4 \mathrm{~mm}$ sclerotic lesion was found within the T8 vertebral body. Brain MRI showed no metastatic lesion and a positron emission tomography (PET) scan showed uptake in the left lung (upper lobe and hilum). She was referred to medical oncology and initiated on chemotherapy with etoposide and cisplatin and radiation therapy for extensive stage small cell cancer of the lung. Most recently, after two cycles of chemotherapy, restaging chest computed tomography (CT) has shown interval improvement in left lung masses.

\section{Discussion}

Metastatic disease to the thyroid is an uncommon diagnosis. Prevalence of metastases to thyroid range between $1 \%$ and $20 \%$, and is generally seen in older populations aged more than 60 years [1]. Metastases to the thyroid are often a marker of poor prognosis [5]. Additionally, studies have also quoted that the incidence of metastases to thyroid is higher in autopsy exams compared to clinical presentations [6]. This is possibly because often the primary disease is quite widespread by the time it involves the thyroid gland. Another reason for this may be due to recent increase in FNAs and biopsies. The most common sites of primary tumors that metastasize to the thyroid include lung, breast, kidney and gastrointestinal tract. Most common lung cancer that spreads to the thyroid is an adenocarcinoma [3]. Other rarer sites of primary tumors that involve the thyroid such as nasopharyngeal carcinoma, choriocarcinoma and osteosarcoma have also been reported [7].

While there are several neuroendocrine tumors found in the thyroid, medullary thyroid cancer (MTC) is the most common one. It originates in the parafollicular cells of the thyroid. It is important to note that calcitonin staining is a characteristic feature of this tumor, thereby making it essential to test in order to make the diagnosis of a secondary tumor in the thyroid. This differentiation is important, as the diagnosis of MTC then leads on to the workup for possible multiple endocrine neoplasia 2 (MEN2). Calcitonin staining on pathology as well as serum calcitonin was negative in our patient. This clarified the diagnosis of the secondary tumor.

Small cell lung cancer is a neuroendocrine tumor of the lung that is characterized by rapid growth. It is generally seen in smokers. The 5-year survival rate for patients with limited disease is about $4.8 \%$ and extensive disease is about $1-2 \%$ [8]. This makes early detection and therapy even more pertinent.

Small cell lung cancer metastasis to the thyroid is rarely reported. Reports in the literature revealed that the diagnosis of the metastatic thyroid lesion was often made at the time of the diagnosis of primary tumor or more commonly in the following few months $[3,9]$. This is in contrast with our patient in whom the thyroid metastasis was the original finding. Additionally, in our patient, the metastasis was multi-centric and only involving one lobe (right side) of the thyroid. It is unclear why the left lobe or isthmus was not involved. Most of the patients, including ours, were clinically euthyroid, although one such patient has also been found to be in thyrotoxicosis [10]. Most patients have a survival of less than 24 months indicating poor prognosis; our patient is currently undergoing treatment and showing good response at 4 months.

Our patient had the total thyroidectomy due to increasing size of goiter and tracheal deviation. She otherwise had no symptoms. This diagnosis was incidentally made on pathology and therefore led to further workup to detect the primary tumor. This alerts clinicians to pay attention to rapidly growing goiters, even in the absence of symptoms. This case report also highlights the unusual presentation of small cell lung cancer with multi-centric metastasis to the thyroid, involving only one lobe. It is unclear why one thyroid lobe would have more affinity to metastatic disease over the other. Very importantly, our patient's case reflects the need to obtain nodule directed FNAs, as the two FNAs done prior to surgery had only revealed lymphocytic thyroiditis. Lastly, a teaching point would be that workup for metastatic disease to the thyroid must be undertaken in a timely fashion, given that most patients have widespread disease by the time of diagnosis. 


\section{Funding}

No funding was applicable to this report.

\section{Conflict of Interest}

The authors report no conflict of interest.

\section{References}

1. Papi G, Fadda G, Corsello SM, Corrado S, Rossi ED, Radighieri E, Miraglia A, et al. Metastases to the thyroid gland: prevalence, clinicopathological aspects and prognosis: a 10-year experience. Clin Endocrinol (Oxf). 2007;66(4):565-571.

2. Baloch ZW, LiVolsi VA. Neuroendocrine tumors of the thyroid gland. Am J Clin Pathol. 2001;115(Suppl):S56-67.

3. Katsenos S, Archondakis S, Vaias M, Skoulikaris N. Thyroid gland metastasis from small cell lung cancer: an unusual site of metastatic spread. J Thorac Dis. 2013;5(2):E21-24.
4. Lin JD, Weng HF, Ho YS. Clinical and pathological characteristics of secondary thyroid cancer. Thyroid. 1998;8(2):149-153.

5. Kim TY, Kim WB, Gong G, Hong SJ, Shong YK. Metastasis to the thyroid diagnosed by fine-needle aspiration biopsy. Clin Endocrinol (Oxf). 2005;62(2):236-241.

6. Wood K, Vini L, Harmer C. Metastases to the thyroid gland: the Royal Marsden experience. Eur J Surg Oncol. 2004;30(6):583-588.

7. Lam KY, Lo CY. Metastatic tumors of the thyroid gland: a study of 79 cases in Chinese patients. Arch Pathol Lab Med. 1998;122(1):37-41.

8. Lassen U, Osterlind K, Hansen M, Dombernowsky P, Bergman B, Hansen HH. Long-term survival in smallcell lung cancer: posttreatment characteristics in patients surviving 5 to $18+$ years - an analysis of 1,714 consecutive patients. J Clin Oncol. 1995;13(5):1215-1220.

9. Can AS, Koksal G. Thyroid metastasis from small cell lung carcinoma: a case report and review of the literature. J Med Case Rep. 2015;9:231.

10. Ozgu ES, Gen R, Ilvan A, Ozge C, Polat A, Vayisoglu Y. Small cell lung cancer with metastasis to the thyroid in a patient with toxic multinodular goiter. J Craniofac Surg. 2012;23(6):e614-615. 